\title{
Hexagonal cell graphs of the normalizer with signature $(2,6, \infty)$
}

\author{
Nazlı Yazıcı Gözütok* (1), Bahadır Özgür Güler (1) \\ Department of Mathematics, Faculty of Science, Karadeniz Technical University, Trabzon, Turkey
}

\begin{abstract}
In this paper, we investigate suborbital graphs $G_{u, n}$ of the normalizer $\Gamma_{B}(N)$ of $\Gamma_{0}(N)$ in $\operatorname{PSL}(2, \mathbb{R})$ for $N=2^{\alpha} 3^{\beta}$, where $\alpha=0,2,4,6$ and $\beta=1,3$. In each of these cases, the normalizer becomes a triangle group and the graph arising from the action of the normalizer contains hexagonal circuits. In order to obtain graphs, we first define an imprimitive action of $\Gamma_{B}(N)$ on $\widehat{\mathbb{Q}}$ using the group $H_{B}(N)$ and then we obtain some properties of the graphs arising from this action.
\end{abstract}

Mathematics Subject Classification (2020). 20H10, 05C25

Keywords. normalizer, suborbital graph, hexagon

\section{Introduction}

Groups are often studied in terms of their actions either on the elements of a set or on particular objects within a structure. One of the most known examples of such situations are maps. A regular map $M$ is a dissection of a closed surface into vertices, edges, and faces such that the automorphism group $\operatorname{Aut}(M)$ acts transitively on vertex-edge-face incidence triples. In fact, they generalize the platonic solids to surfaces other than the sphere. Having the "highest level of symmetry", the theory of maps and their symmetries is surprisingly rich and interacts with other disciplines in mathematics such as algebraic topology, group theory, hyperbolic geometry, the theory of Riemann surfaces, and Galois theory. We refer the reader to $[6,7,12]$ for further reading.

Understanding groups like $\Gamma_{0}(N)$ or other congruence groups of the modular group and their normalizers in $P S L(2, \mathbb{R})$ are of special interest for finite group theorists due their links with the Monster simple group. Our object in the present paper is one of them, the normalizer of $\Gamma_{0}(N)$ in $P S L(2, \mathbb{R})$.

As indicated in [19], the existence of infinitely many maps with given properties often reduces to related triangle groups. With this motivation, we have already examined the relationship between the normalizer with signatures $(2,3, \infty)$ and $(2,4, \infty)$, and the regular maps in [20,21], respectively. The work in this paper may be thought of as a continuation of work done in [20,21]. Although, many of the results and their proofs in this paper are straightforward adjustments of those in [20,21], our purpose here is to investigate

\footnotetext{
${ }^{*}$ Corresponding Author.

Email addresses: nazliyazici@ktu.edu.tr (N. Yazıcı Gözütok), boguler@ktu.edu.tr (B.Ö. Güler)

Received: 11.11.2020; Accepted: 20.11.2021
} 
some combinatorial properties of the normalizer with signature $(2,6, \infty)$ by a suitable map subgroup. Lastly, we also refer the reader to $[3,9-11,16-18]$ for the relationship of the principal congruence subgroup $\bmod n$ of the Modular group and regular maps in this manner.

\section{Preliminaries}

Let $\operatorname{PSL}(2, \mathbb{R})$ denote the group of all linear fractional transformation

$$
T: z \rightarrow \frac{a z+b}{c z+d} \text {, where } a, b, c \text { and } d \text { are reals with } a d-b c=1 .
$$

In terms of matrix representation, the elements of $P S L(2, \mathbb{R})$ correspond to the matrices

$$
\pm\left(\begin{array}{ll}
a & b \\
c & d
\end{array}\right), \text { where } a, b, c \text { and } d \text { are reals with } a d-b c=1 .
$$

This is in fact the automorphism group of the upper half plane

$$
\mathbb{H}:=\{z \in \mathbb{C}: \operatorname{Im}(\mathrm{z})>0\} .
$$

The modular group $\Gamma$ is the subgroup of $P S L(2, \mathbb{R})$ such that $a, b, c, d$ are integers. The subgroup $\Gamma_{0}(N)=\{g \in \Gamma: c \equiv 0(\bmod N)\}$ is a well-known congruence subgroup of the classical modular group $\Gamma$. The normalizer of $\Gamma_{0}(N)$ in $P S L(2, \mathbb{R})$ turns to be a very important group in the study of moonshine and for this reason has been studied by many authors (see, for example, $[4,5,8,13,14])$. It consists exactly of the matrices

$$
\left(\begin{array}{cc}
a e & b / h \\
c N / h & d e
\end{array}\right)
$$

where $e \| \frac{N}{h^{2}}$ and $h$ is the largest divisor of 24 for which $h^{2} \mid N$. Hence, the determinant e of the matrix is positive, and $r \| s$ means that $r \mid s$ and $(r, s / r)=1$ ( $r$ is called an exact divisor of $s)$. We denote the normaliser by $\Gamma_{B}(N)$.

\section{The action of $\Gamma_{B}(N)$ on $\hat{\mathbb{Q}}$}

From now on, unless otherwise stated explicitly, $N$ denotes an integer such that $N=$ $2^{\alpha} 3^{\beta}$ for $\alpha=0,2,4,6$ and $\beta=1,3$. For these values of $N$, we have $3 h^{2}=N$ and $e=1$ or 3 , so the group $\Gamma_{B}(N)$ is the set of transformations corresponding to the matrices

I.

II.

$$
\left(\begin{array}{cc}
a & b / h \\
3 c h & d
\end{array}\right), \quad a d-3 b c=1
$$

$$
\left(\begin{array}{cc}
3 a & b / h \\
3 c h & 3 d
\end{array}\right), \quad 9 a d-3 b c=3 \text { or } 3 a d-b c=1,
$$

where $h$ is the largest divisor of 24 for which $h^{2} \mid N$.

Definition 3.1. The elements of $\Gamma_{B}(N)$ of type I is called even elements and the elements of type II is called odd elements. The set of all even elements is denoted by $H_{B}(N)$.

Proposition 3.2. $H_{B}(N)$ is a subgroup of index 2 in $\Gamma_{B}(N)$.

Proof. It is clear that $H_{B}(N) \leq \Gamma_{B}(N)$. We show that the index is 2 . In order to do this, we show that any two odd elements of $\Gamma_{B}(N)$ determine the same coset of $H_{B}(N)$ in $\Gamma_{B}(N)$. Let

$$
A_{1}=\left(\begin{array}{cc}
3 a_{1} & b_{1} / h \\
3 c_{1} h & 3 d_{1}
\end{array}\right), \quad A_{2}=\left(\begin{array}{cc}
3 a_{2} & b_{2} / h \\
3 c_{2} h & 3 d_{2}
\end{array}\right)
$$


be two odd elements. Then $A_{1}$ and $A_{2}$ determine the same coset of $H_{B}(N)$ in $\Gamma_{B}(N)$ if and only if $A_{2}^{-1} A_{1} \in H_{B}(N)$. Thus, it is enough to show that $A_{2}^{-1} A_{1} \in H_{B}(N)$. To this end, we have

$$
\begin{aligned}
A_{2}^{-1} A_{1} & =\left(\begin{array}{cc}
3 d_{2} & -b_{2} / h \\
-3 c_{2} h & 3 a_{2}
\end{array}\right)\left(\begin{array}{cc}
3 a_{1} & b_{1} / h \\
3 c_{1} h & 3 d_{1}
\end{array}\right) \\
& =\left(\begin{array}{cc}
9 a_{1} d_{2}-3 c_{1} b_{2} & 3\left(b_{1} d_{2}-b_{2} d_{1}\right) / h \\
9\left(a_{2} c_{1}-c_{2} a_{1}\right) h & 9 d_{2} a_{1}-3 b_{1} c_{2}
\end{array}\right) .
\end{aligned}
$$

The resulting matrix has determinant 9 , thus if we divide all entries by 3 , transformation does not change and we have determinant equal to 1 . Thus, by the definition of an even element, we have

$$
A_{2}^{-1} A_{1}=\left(\begin{array}{cc}
3 a_{1} d_{2}-c_{1} b_{2} & \left(b_{1} d_{2}-b_{2} d_{1}\right) / h \\
3\left(a_{2} c_{1}-c_{2} a_{1}\right) h & 3 d_{2} a_{1}-b_{1} c_{2}
\end{array}\right) \in H_{B}(N)
$$

Now, we determine the action of $\Gamma_{B}(N)$ on $\widehat{\mathbb{Q}}$. Every element of $\widehat{\mathbb{Q}}$ can be represented by a reduced fraction $\frac{x}{y}$, with $x, y \in \mathbb{Z}$ and $(x, y)=1$. Since $\frac{x}{y}=\frac{-x}{-y}$, this representation is not unique. We represent $\infty$ as $\frac{1}{0}=\frac{-1}{0}$. The action of the transformation $\left(\begin{array}{ll}a & b \\ c & d\end{array}\right)$ on $\frac{x}{y}$ is

$$
\left(\begin{array}{ll}
a & b \\
c & d
\end{array}\right): \frac{x}{y} \rightarrow \frac{a x+b y}{c x+d y}
$$

Note that if $\left(\begin{array}{ll}a & b \\ c & d\end{array}\right)$ has determinant 1 and $(x, y)=1$, then $(a x+b y, c x+d y)=1$.

The following theorem holds by [1] as a consequence of cusp number. Here, we give a different arithmetical proof which more suitable for our purpose.

Theorem 3.3. The action of $\Gamma_{B}(N)$ on $\widehat{\mathbb{Q}}$ is transitive.

Proof. It is enough to prove that the orbit of $\infty$ is $\widehat{\mathbb{Q}}$. Let $\frac{x}{y} \in \widehat{\mathbb{Q}}$. There are three cases to be considered:

Case 1. Assume that $(y, h)=1$.

i) Let $3 \mid y$. In this case, there is an integer $k$ such that $y=3 k$. Since $(x, y)=1$, $(h x, y)=1$. Then there exist integers $a, b$ such that $a h x-b y=1$. Now consider the matrix $\left(\begin{array}{cc}h x & b / h \\ h y & a\end{array}\right)$. Determinant of this matrix is 1 and by using $y=3 k$, we have

$$
\left(\begin{array}{cc}
h x & b / h \\
h y & a
\end{array}\right)=\left(\begin{array}{cc}
h x & b / h \\
3 h k & a
\end{array}\right) \in \Gamma_{B}(N)
$$

and

$$
\left(\begin{array}{cc}
h x & b / h \\
h y & a
\end{array}\right)\left(\begin{array}{l}
1 \\
0
\end{array}\right)=\frac{x}{y}
$$

ii) Let $3 \nmid y$. Since $(x, y)=1,(h x, y)=1$. Also, as $y$ is odd, we have $(3 h x, y)=1$. Thus there exist integers $a, b$ such that $3 a h x-b y=1$. Now consider the matrix $\left(\begin{array}{cc}3 h x & b / h \\ 3 h y & 3 a\end{array}\right)$. Determinant of this matrix is 3 . Thus we have 


$$
\left(\begin{array}{cc}
3 h x & b / h \\
3 h y & 3 a
\end{array}\right) \in \Gamma_{B}(N)
$$

and

$$
\left(\begin{array}{cc}
3 h x & b / h \\
3 h y & 3 a
\end{array}\right)\left(\begin{array}{l}
1 \\
0
\end{array}\right)=\frac{x}{y} .
$$

Case 2. Assume that $(y, h)=h$. In this case, as $h \mid y$, there is an integer $t$ such that $y=h t$.

i) Let $3 \nmid y$. Since $3 \nmid y$ and $(x, y)=1$, we have $(3 x, y)=1$. Thus there exist integers $a, b$ such that $3 a x-b y=1$. Now consider the matrix $\left(\begin{array}{cc}3 x & b \\ 3 y & 3 a\end{array}\right)$. Determinant of this matrix is 3 and by using $y=h t$, we have

$$
\left(\begin{array}{cc}
3 x & b \\
3 y & 3 a
\end{array}\right)=\left(\begin{array}{cc}
3 x & b h / h \\
3 t h & 3 a
\end{array}\right) \in \Gamma_{B}(N)
$$

and

$$
\left(\begin{array}{cc}
3 x & b \\
3 y & 3 a
\end{array}\right)\left(\begin{array}{l}
1 \\
0
\end{array}\right)=\frac{x}{y}
$$

ii) Let $3 \mid y$. We know that $y=h t$. Also, $t$ could satisfy $3 \mid t$ or $3 \nmid t$.

A) Let $3 \mid t$. In this case, there is an integer $k$ such that $t=3 k$. Substituting this in $y=h t$, we have $y=3 h k$. Since $(x, y)=1$, there exist integers $a, b$ such that $a x-b y=1$. Now consider the matrix $\left(\begin{array}{ll}x & b \\ y & a\end{array}\right)$. Determinant of this matrix is 1 and by using $y=3 h k$, we have

$$
\left(\begin{array}{ll}
x & b \\
y & a
\end{array}\right)=\left(\begin{array}{cc}
x & b h / h \\
3 k h & a
\end{array}\right) \in \Gamma_{B}(N)
$$

and

$$
\left(\begin{array}{ll}
x & b \\
y & a
\end{array}\right)\left(\begin{array}{l}
1 \\
0
\end{array}\right)=\frac{x}{y}
$$

B) Let $3 \nmid t$. As $(x, y)=1,(x, t)=1$. Also, as $3 \nmid t$, it is obtained $(3 x, t)=1$. Thus there exist integers $a, b$ such that $3 a x-b t=1$. Now consider the matrix $\left(\begin{array}{cc}3 x & b / h \\ 3 y & 3 a\end{array}\right)$. Determinant of this matrix is 3 and by using $y=h t$, we have

$$
\left(\begin{array}{cc}
3 x & b / h \\
3 y & 3 a
\end{array}\right)=\left(\begin{array}{cc}
3 x & b / h \\
3 t h & 3 a
\end{array}\right) \in \Gamma_{B}(N)
$$

and

$$
\left(\begin{array}{cc}
3 x & b / h \\
3 y & 3 a
\end{array}\right)\left(\begin{array}{l}
1 \\
0
\end{array}\right)=\frac{x}{y}
$$

Case 3. Assume that $(h, y)=s$, where $s \neq 1$ and $s \neq h$. Since $s \mid h$ and $s \mid y$, there exist integer $m, n$ such that $h=s m, y=s n$ and $(m, n)=1$.

i) Let $3 \nmid t$. Since $(m, y)=1$ and $(x, y)=1,(m x, y)=1$. Also, as $3 \nmid t$, we have $(3 m x, y)=1$. Thus there exist integers $a, b$ such that $3 a m x-b y=1$. Now consider the matrix $\left(\begin{array}{cc}3 m x & b / m \\ 3 m y & 3 a\end{array}\right)$. Determinant of this matrix is 3 and by using $h=s m$ and $y=s n$, we have 


$$
\left(\begin{array}{cc}
3 m x & b / m \\
3 n h & 3 a
\end{array}\right)=\left(\begin{array}{cc}
3 m x & b s / h \\
3 n h & 3 a
\end{array}\right) \in \Gamma_{B}(N)
$$

and

$$
\left(\begin{array}{cc}
3 m x & b / m \\
3 m y & 3 a
\end{array}\right)\left(\begin{array}{l}
1 \\
0
\end{array}\right)=\frac{x}{y}
$$

ii) Let $3 \mid y$. We know that $y=k n$. Also, $n$ could satisfy $3 \mid n$ or $3 \nmid n$.

A) Let $3 \mid n$. There is an integer $t$ such that $n=3 t$. Thus, we obtain $y=3$ st. Since $3 \mid t$ and $(x, y)=1$, we have $3 \nmid x$. Similarly as $3 \mid n$ and $(m, n)=1$, we have $3 \nmid m$. On the other hand, $(x, t)=1$ and $3 \nmid x$, and so, $(x, 3 t)=1$. Similarly, since $(m, n)=1$, we have $(m, 3 t)=1$. Thus $(x, 3 t)=1$ and $(m, 3 t)=1$ yield $(m x, 3 t)=1$. Then there exist integers $a, b$ such that $a m x-3 b t=1$. Now consider the matrix $\left(\begin{array}{cc}m x & b / h \\ 3 t h & a\end{array}\right)$. Determinant of this matrix is 1 , then we have

$$
\left(\begin{array}{cc}
m x & b / h \\
3 t h & a
\end{array}\right) \in \Gamma_{B}(N)
$$

and

$$
\left(\begin{array}{cc}
m x & b / h \\
3 t h & a
\end{array}\right)\left(\begin{array}{l}
1 \\
0
\end{array}\right)=\frac{x}{y} .
$$

B) Let $3 \nmid n$. Note that $(x, y)=1$ yields $(x, n)=1$. As $3 \nmid n,(3 x, n)=1$. Also using $(m, n)=1$, we have $(3 m x, n)=1$. Thus there exist integers $a, b$ such that $3 a m x-b n=1$. Now consider the matrix $\left(\begin{array}{cc}3 m x & b / h \\ 3 n h & 3 a\end{array}\right)$. Determinant of this matrix is 3 , thus

$$
\left(\begin{array}{cc}
3 m x & b / h \\
3 n h & 3 a
\end{array}\right) \in \Gamma_{B}(N)
$$

and

$$
\left(\begin{array}{cc}
3 m x & b / h \\
3 n h & 3 a
\end{array}\right)\left(\begin{array}{l}
1 \\
0
\end{array}\right)=\frac{x}{y}
$$

Theorem 3.4. The stabiliser of $\infty$ in $\Gamma_{B}(N)$ is the group whose elements are the transformations corresponding to the matrices

$$
\left(\begin{array}{cc}
1 & u / h \\
0 & 1
\end{array}\right), u \in \mathbb{Z}
$$

Proof. First we show that an odd element of $\Gamma_{B}(N)$ does not stabilise $\infty$. If it does, we have

$$
\left(\begin{array}{cc}
3 a & b / h \\
3 c h & 3 d
\end{array}\right)\left(\begin{array}{l}
1 \\
0
\end{array}\right)=\left(\begin{array}{l}
1 \\
0
\end{array}\right) .
$$

This yields $a=1$ and $c=0$. By determinant, it is written $3 a d-b c=1$. Substituting $a=1$ and $c=0$ in $3 a d-b c=1$ yields $3 d=1$. This contradicts with $d$ is an integer. Thus, the elements of $\Gamma_{B}(N)$ stabilising $\infty$ are even elements. Now, for an even element stabilising $\infty$,

$$
\left(\begin{array}{cc}
a & b / h \\
3 c h & d
\end{array}\right)\left(\begin{array}{l}
1 \\
0
\end{array}\right)=\left(\begin{array}{l}
1 \\
0
\end{array}\right) .
$$


It is obtained $a=1$ and $c=0$. By determinant we have also $d=1$. Therefore an even element stabilising $\infty$ is of the form $\left(\begin{array}{cc}1 & b / h \\ 0 & 1\end{array}\right)$.

Conversely, since $\left(\begin{array}{cc}1 & u / h \\ 0 & 1\end{array}\right)\left(\begin{array}{l}1 \\ 0\end{array}\right)=\left(\begin{array}{l}1 \\ 0\end{array}\right)$, any element that has the form $\left(\begin{array}{cc}1 & u / h \\ 0 & 1\end{array}\right)$ is contained in the stabiliser of $\infty$.

Now we consider the imprimitivity of the action of $\Gamma_{B}(N)$ on $\widehat{\mathbb{Q}}$, hence we start with a general discussion of primitivity of permutation groups. Let $(G, \Delta)$ be a transitive permutation group, consisting of a group $G$ acting on a set $\Delta$ transitively. An equivalence relation $\approx$ on $\Delta$ is called $G$-invariant if, whenever $\alpha, \beta \in \Delta$ satisfy $\alpha \approx \beta$, then $g(\alpha) \approx g(\beta)$ for all $g \in G$. The equivalence classes are called blocks, and the block containing $\alpha$ is denoted by $[\alpha]$. We call $(G, \Delta)$ imprimitive if $\Delta$ admits some $G$-invariant equivalence relation different from

i) the identity relation, that is, $\alpha \approx \beta$ if and only if $\alpha=\beta$;

ii) the universal relation, that is, $\alpha \approx \beta$ for all $\alpha, \beta \in \Delta$.

Otherwise $(G, \Delta)$ is called primitive. These two relations are supposed to be trivial relations. Clearly, a primitive group must be transitive, for if not the orbits would form a system of blocks. The converse is false, but we have the following useful result.

Lemma 3.5 ([2], Chapter 1, Theorem 1.6.5). Let $(G, \Delta)$ be a transitive permutation group. Then $(G, \Delta)$ is primitive if and only if the stabilizer $G_{\alpha}$ of $\alpha \in \Delta$ is a maximal subgroup of $G$ for each $\alpha \in \Delta$.

By the above lemma we see that whenever for some $\alpha, G_{\alpha} \lesseqgtr H \lesseqgtr G$, then $\Delta$ admits some $G$-invariant equivalence relations other than the trivial cases. Because of the transitivity, every element of $\Delta$ has the form $g(\alpha)$ for some $g \in G$. Thus one of the non-trivial $G$-invariant equivalence relations on $\Delta$ is given as follows:

$$
g(\alpha) \approx g^{\prime}(\alpha) \text { if and only if } g^{\prime} \in g H .
$$

If we set $G=\Gamma_{B}(N), \Delta=\widehat{\mathbb{Q}}, H=H_{B}(N)$, the set of even elements of $\Gamma_{B}(N)$, and $G_{\alpha}=\Gamma_{B}(N)_{\infty}$ the stabiliser of $\infty$ in $\Gamma_{B}(N)$, then we clearly see that $\Gamma_{B}(N)_{\infty} \lesseqgtr H_{B}(N) \lesseqgtr$ $\Gamma_{B}(N)$. Thus, by Lemma $3.5, \Gamma_{B}(N)$ acts imprimitively on $\widehat{\mathbb{Q}}$.

We define the following $\Gamma_{B}(N)$-invariant equivalence relation $\approx$ on $\widehat{\mathbb{Q}}$. Since $\Gamma_{B}(N)$ acts transitively on $\widehat{\mathbb{Q}}$, every element of $\widehat{\mathbb{Q}}$ has the form $g(\infty)$ for some $g \in \Gamma_{B}(N)$. Therefore, it is easily seen that

$$
g(\infty) \approx g^{\prime}(\infty) \text { if and only if } g^{\prime} \in g H_{B}(N)
$$

gives a $\Gamma_{B}(N)$-invariant imprimitive equivalence relation.

By our general discussion of imprimitivity, the number of blocks under $\approx$ is given by the index $\left|\Gamma_{B}(N): H_{B}(N)\right|$. By Proposition 3.2, the number of blocks is 2 . It is easily seen that the blocks are

$$
\begin{aligned}
& {[0]=\left\{\frac{x}{y h}:(x, y)=1 \text { and } 3 \nmid y\right\}} \\
& {[\infty]=\left\{\frac{x}{3 y h}:(x, y)=1 \text { and } 3 \nmid x\right\} .}
\end{aligned}
$$

This result shows that there are two types of vertices in $\widehat{\mathbb{Q}}$. We call the vertices in $[0]$, the odd vertices, and the vertices in [ $\infty]$, the even vertices. For instance, by considering the $\infty$ as $\frac{1}{3.0 . h}$, we have $\infty$ is an even vertex whereas by considering 0 as $\frac{0}{1 . h}$, we have 0 is an odd vertex. 


\section{Suborbital graphs for $\Gamma_{B}(N)$}

A suborbital graph is a graph arisen from a transitive group action. The concept of this graph was introduced by Sims in [15]. It is known that a map is an embedding of a graph into a surface, and the faces of this map is formed by the circuits of the graph. In this section we aim to find hexagonal circuits using suborbital graphs.

Let $(G, \Delta)$ be a transitive permutation group. Then $G$ acts on $\Delta \times \Delta$ by

$$
T:(\alpha, \beta) \mapsto(T(\alpha), T(\beta)), T \in G, \alpha, \beta \in \Delta .
$$

The orbits of this action are called suborbitals of $G$, that contained $\alpha, \beta$ and are denoted by $\mathcal{O}(\alpha, \beta)$. For $\mathcal{O}(\alpha, \beta)$ we can form a suborbital graph $G(\alpha, \beta)$. Its vertices are the elements of $\Delta$ and there is an edge from $\gamma$ to $\delta$, denoted by $\gamma \rightarrow \delta$, if $(\gamma, \delta) \in G(\alpha, \beta)$. A circuit of length $m$ is a sequence $\nu_{1} \rightarrow \nu_{2} \rightarrow \cdots \rightarrow \nu_{m} \rightarrow \nu_{1}$ such that $\nu_{i} \neq \nu_{j}$ for $i \neq j$, where $m \geq 3$. We call a circuit a triangle, a quadrilateral or a hexagon if $m=3,4$ or $m=6$, respectively.

Indeed $\mathcal{O}(\beta, \alpha)$ is also a suborbital. This suborbital is either equal to or disjointed from $\mathcal{O}(\alpha, \beta)$. If orbitals are disjoint, then $G(\beta, \alpha)$ is just $G(\alpha, \beta)$ with the arrows reversed. In this case, the suborbital graphs $G(\beta, \alpha)$ and $G(\alpha, \beta)$ are called paired. If orbitals are equal, then we have $G(\beta, \alpha)=G(\alpha, \beta)$. Then the suborbital graph $G(\alpha, \beta)$ is called self-paired.

As $\Gamma_{B}(N)$ acts transitively on $\widehat{\mathbb{Q}}$, then every suborbital contains a pair $\left(\infty, \frac{u}{n}\right)$ for some $\frac{u}{n} \in \widehat{\mathbb{Q}}$. This suborbital is denoted by $\mathcal{O}_{u, n}$ and the corresponding suborbital graph is denoted by $G_{u, n}$. We now investigate some properties of $G_{u, n}$.

Lemma 4.1. Let $\frac{u}{n} \in \widehat{\mathbb{Q}},(n, h)=t$ and $n=n_{1} t, h=h_{1} t$. Also, $A=\left(\begin{array}{cc}a & b / h \\ 3 c h & d\end{array}\right)$, and $B=\left(\begin{array}{cc}3 a^{\prime} & b^{\prime} / h \\ 3 c^{\prime} h & 3 d^{\prime}\end{array}\right) \in \Gamma_{B}(N)$. Then the followings hold:

i) If $3 \mid n_{1}$, then $A\left(\frac{u}{n}\right)$ is an even vertex and $B\left(\frac{u}{n}\right)$ is an odd vertex.

ii) If $3 \nmid n_{1}$, then $A\left(\frac{u}{n}\right)$ is an odd vertex and $B\left(\frac{u}{n}\right)$ is an even vertex.

Proof. First, let us calculate the actions of $A$ and $B$ on $\frac{u}{n}$ :

$$
\begin{gathered}
A\left(\frac{u}{n}\right)=\left(\begin{array}{cc}
a & b / h \\
3 c h & d
\end{array}\right)\left(\begin{array}{l}
u \\
n
\end{array}\right)=\frac{a u h+b n}{(3 c u h+d n) h}=\frac{a u h_{1}+b n_{1}}{\left(3 c u h_{1}+d n_{1}\right) h}, \\
B\left(\frac{u}{n}\right)=\left(\begin{array}{cc}
3 a^{\prime} & b^{\prime} / h \\
3 c^{\prime} h & 3 d^{\prime}
\end{array}\right)\left(\begin{array}{l}
u \\
n
\end{array}\right)=\frac{3 a^{\prime} u h+b^{\prime} n}{3\left(c^{\prime} u h+d^{\prime} n\right) h}=\frac{3 a^{\prime} u h_{1}+b^{\prime} n_{1}}{3\left(c^{\prime} u h_{1}+d^{\prime} n_{1}\right) h} .
\end{gathered}
$$

Now let $3 \mid n_{1}$. Then there is an integer $n_{2}$ such that $n_{1}=3 n_{2}$. By substituting this in (4.1), we have

$$
A\left(\frac{u}{n}\right)=\frac{a u h_{1}+b n_{1}}{3\left(c u h_{1}+d n_{2}\right) h} .
$$

Since the determinant of $A$ is $1,3 \nmid a$. Also, since $3 \mid n_{1}$ and $\left(u, n_{1}\right)=1,\left(n_{1}, h_{1}\right)=1$, it is obtained that $3 \nmid u$, and $3 \nmid h_{1}$. Therefore the expression in the numerator of $A\left(\frac{u}{n}\right)$ does not divide 3. Moreover, the matrix $\left(\begin{array}{cc}a & b \\ 3 c & d\end{array}\right)$ has determinant 1 and $\left(u h_{1}, n_{1}\right)=1$. Consider the action

$$
\left(\begin{array}{cc}
a & b \\
3 c & d
\end{array}\right)\left(\begin{array}{c}
u h_{1} \\
n_{1}
\end{array}\right)=\frac{a u h_{1}+b n_{1}}{3 c u h_{1}+d n 1}=\frac{a u h_{1}+b n_{1}}{3\left(c u h_{1}+d n_{2}\right)} .
$$


Thus, we have $\left(a u h_{1}+b n_{1}, 3\left(c u h_{1}+d n_{2}\right)\right)=1$. Giving $\left(a u h_{1}+b n_{1}, c u h_{1}+d n_{2}=1\right)=1$. By the definition of an even vertex, $A\left(\frac{u}{n}\right)$ is an even vertex.

For $B\left(\frac{u}{n}\right)$, by substituting $n_{1}=3 n_{2}$ in (4.2), we have

$$
B\left(\frac{u}{n}\right)=\frac{3\left(a^{\prime} u h_{1}+b^{\prime} n_{2}\right)}{3\left(c^{\prime} u h_{1}+2 d^{\prime} n_{2}\right) h}=\frac{a^{\prime} u h_{1}+b^{\prime} n_{2}}{\left(c^{\prime} u h_{1}+3 d^{\prime} n_{2}\right) h} .
$$

Since the determinant of $A$ is $1,3 \nmid c^{\prime}$. Also, since $3 \mid n_{1}$ and $\left(u, n_{1}\right)=1,\left(n_{1}, h_{1}\right)=1$, it is obtained that $u$ and $h_{1}$ are odd. Note that $3 \nmid c^{\prime} u h_{1}+3 d^{\prime} n_{2}$. Moreover, the matrix $\left(\begin{array}{cc}3 a & b \\ c & d\end{array}\right)$ has determinant 1 and $\left(u h_{1}, n_{2}\right)=1$. Consider the action

$$
\left(\begin{array}{cc}
a^{\prime} & b^{\prime} \\
c^{\prime} & 3 d^{\prime}
\end{array}\right)\left(\begin{array}{c}
u h_{1} \\
n_{2}
\end{array}\right)=\frac{a^{\prime} u h_{1}+b^{\prime} n_{2}}{c^{\prime} u h_{1}+3 d^{\prime} n_{2}}
$$

Thus, we have $\left(a^{\prime} u h_{1}+b^{\prime} n_{2}, c^{\prime} u h_{1}+3 d^{\prime} n_{2}\right)=1$. By the definition of an odd vertex, $B\left(\frac{u}{n}\right)$ is an odd vertex.

The proof of $(i i)$ is similar to the proof of $(i)$.

Theorem 4.2 (Edge Conditions). Let $\frac{u}{n} \in \widehat{\mathbb{Q}},(n, h)=t$ and $n=n_{1} t, h=h_{1} t$. Then the followings hold:

i) If $3 \mid n_{1}$, then

I) there is an edge $\frac{r}{3 s h} \rightarrow \frac{x}{3 y h}$ in $G_{u, n}$ if and only if $x \equiv \pm$ urh $h_{1}\left(\bmod n_{1}\right)$, $y \equiv \pm u s h_{1}\left(\bmod \frac{n_{1}}{3}\right)$ and $r y-s x= \pm \frac{n_{1}}{3}$.

II) There is an edge $\frac{r}{s h} \rightarrow \frac{x}{y h}$ in $G_{u, n}$ if and only if $x \equiv \pm u r h_{1}\left(\bmod \frac{n_{1}}{3}\right)$, $y \equiv \pm u s h_{1}\left(\bmod n_{1}\right)$ and $r y-s x= \pm \frac{n_{1}}{3}$.

III) There are no edges of the form $\frac{r}{3 s h} \rightarrow \frac{{ }^{x}}{y h}$ and $\frac{r}{s h} \rightarrow \frac{x}{3 y h}$ in $G_{u, n}$.

ii) If $3 \nmid n_{1}$,

I) There is an edge $\frac{r}{s h} \rightarrow \frac{x}{3 y h}$ in $G_{u, n}$ if and only if $x \equiv \pm 3$ urh $h_{1}\left(\bmod n_{1}\right)$, $y \equiv \pm u s h_{1}\left(\bmod n_{1}\right)$ and $3 r y-s x= \pm n_{1}$.

II) There is an edge $\frac{r}{3 s h} \rightarrow \frac{x}{y h}$ in $G_{u, n}$ if and only if $x \equiv \pm$ urh $h_{1}\left(\bmod n_{1}\right)$, $y \equiv \pm 3 u s h_{1}\left(\bmod n_{1}\right)$ and $r y-3 s x= \pm n_{1}$.

III) There are no edges of the form $\frac{r}{3 s h} \rightarrow \frac{x}{3 y h}$ and $\frac{r}{s h} \rightarrow \frac{x}{y h}$ in $G_{u, n}$.

Proof. We prove only (i). The proof of $(i i)$ is similar. Let $3 \mid n_{1}$.

I) We show only the case $\frac{r}{3 s h} \rightarrow \frac{x}{3 y h}$; the other case is similar. Suppose that there is an edge $\frac{r}{3 s h} \rightarrow \frac{x}{3 y h}$ in $G_{u, n}$. Then there exists an element $T$ in $\Gamma_{B}(N)$ such that $T\left(\frac{1}{0}\right)=\frac{r}{3 s h}$ and $T\left(\frac{u}{n}\right)=\frac{x}{3 y h}$. Since $T\left(\frac{1}{0}\right)=\frac{r}{3 s h}, T$ is an even element. Thus, if $T=\left(\begin{array}{cc}a & b / h \\ 3 c h & d\end{array}\right)$, we have

$$
T\left(\frac{1}{0}\right)=\left(\begin{array}{cc}
a & b / h \\
3 c h & d
\end{array}\right)\left(\begin{array}{l}
1 \\
0
\end{array}\right)=\frac{a}{3 c h}=\frac{r}{3 s h},
$$


which yields $a=r$ and $c=s$. On the other hand,

$$
T\left(\frac{u}{n}\right)=\left(\begin{array}{cc}
a & b / h \\
3 c h & d
\end{array}\right)\left(\begin{array}{l}
u \\
n
\end{array}\right)=\frac{a u h+b n}{(3 c u h+d n) h}=\frac{a u h_{1}+d n_{1}}{3\left(c u h_{1}+d \frac{n_{1}}{2}\right) h}=\frac{x}{3 y h}
$$

gives us $x=a u h_{1}+d n_{1}$ and $y=c u h_{1}+d \frac{n_{1}}{3}$. Using the last equations, and, $a=r$ and $c=s$, we conclude that $x \equiv u r h_{1}\left(\bmod n_{1}\right), y \equiv u s h_{1}\left(\bmod \frac{n_{1}}{3}\right)$. Also, by the determinant of $T$, we know that $a d-3 b c=1$. Note that

$$
\left(\begin{array}{cc}
a h & b \\
3 c h & d
\end{array}\right)\left(\begin{array}{ll}
1 & u \\
0 & n
\end{array}\right)=\left(\begin{array}{cc}
a h & a u h+b n \\
3 c h & 3 c u h+d n
\end{array}\right)=\left(\begin{array}{cc}
r h & t x \\
3 s h & 3 t y
\end{array}\right) .
$$

The determinants of the matrices on the left hand side of the above equation are $h$ and $n$, respectively. Also, the determinant of the matrix on the right hand side is $3 t h(r y-s x)$. Thus, we have $h n=3 t h(r y-s x)$ and so $r y-s x=\frac{n_{1}}{3}$.

Conversely, let $x \equiv u_{u r h}\left(\bmod n_{1}\right), y \equiv u s h_{1}\left(\bmod \frac{n_{1}}{3}\right)$ and $r y-s x=\frac{n_{1}}{3}$. Since $x \equiv u r h_{1}\left(\bmod n_{1}\right), y \equiv u s h_{1}\left(\bmod \frac{n_{1}}{3}\right)$, there exist integers $b, d$ such that $x=u r h_{1}+b n_{1}$ and $y=u s h_{1}+d \frac{n_{1}}{3}$. Consider the matrix $S=\left(\begin{array}{cc}r & b / h \\ 3 s h & d\end{array}\right)$. As $r y-s x=\frac{n_{1}}{3}$, it is obtained that $r d-3 b s=1$. This yields $S \in \Gamma_{B}(N)$. One can easily verify that $S\left(\frac{1}{0}\right)=\frac{r}{3 s h}$ and $S\left(\frac{u}{n}\right)=\frac{x}{3 y h}$. Thus $\frac{r}{3 s h} \rightarrow \frac{x}{3 y h}$ is an edge in $G_{u, n}$.

II) We show only the case $\frac{r}{s h} \rightarrow \frac{x}{y h}$; the other case is similar. Suppose that there is an edge $\frac{r}{s h} \rightarrow \frac{x}{y h}$ in $G_{u, n}$. Then there exists an element $T$ in $\Gamma_{B}(N)$ such that $T\left(\frac{1}{0}\right)=\frac{r}{s h}$ and $T\left(\frac{u}{n}\right)=\frac{x}{y h}$. Since $T\left(\frac{1}{0}\right)=\frac{r}{s h}, T$ is an odd element. Thus, if $T=\left(\begin{array}{cc}3 a & b / h \\ 3 c h & 3 d\end{array}\right)$, we have

$$
T\left(\frac{1}{0}\right)=\left(\begin{array}{cc}
3 a & b / h \\
3 c h & 3 d
\end{array}\right)\left(\begin{array}{l}
1 \\
0
\end{array}\right)=\frac{a}{c h}=\frac{r}{s h},
$$

which yields $a=r$ and $c=s$. On the other hand,

$$
\begin{aligned}
T\left(\frac{u}{n}\right) & =\left(\begin{array}{cc}
3 a & b / h \\
3 c h & 3 d
\end{array}\right)\left(\begin{array}{l}
u \\
n
\end{array}\right)=\frac{3 a u h+b n}{3(c u h+d n) h} \\
& =\frac{3\left(a u h_{1}+d \frac{n_{1}}{3}\right)}{3\left(c u h_{1}+d n_{1}\right) h}=\frac{a u h_{1}+d \frac{n_{1}}{3}}{\left(c u h_{1}+d n_{1}\right) h} \frac{x}{y h}
\end{aligned}
$$

gives us $x=a u h_{1}+d \frac{n_{1}}{3}$ and $y=c u h_{1}+d n_{1}$. Using the last equations, and, $a=r$ and $c=s$, we conclude that $x \equiv u r h_{1}\left(\bmod \frac{n_{1}}{3}\right), y \equiv u s h_{1}\left(\bmod n_{1}\right)$. Also, by the determinant of $T$, we know that $3 a d-b c=1$. Note that

$$
\left(\begin{array}{cc}
3 a h & b \\
3 c h & 3 d
\end{array}\right)\left(\begin{array}{cc}
1 & u \\
0 & n
\end{array}\right)=\left(\begin{array}{cc}
3 a h & 3 a u h+b n \\
3 c h & 3 c u h+2 d n
\end{array}\right)=\left(\begin{array}{cc}
3 r h & 3 t x \\
3 s h & 3 t y
\end{array}\right) .
$$

The determinants of the matrices on the left hand side of the above equation are $3 h$ and $n$, respectively. Also, the determinant of the matrix on the right hand side is $9 \operatorname{th}(r y-s x)$. Thus, we have $3 h n=9 \operatorname{th}(r y-s x)$ and so $r y-s x=\frac{n_{1}}{3}$. 
Conversely, let $x \equiv u r h_{1}\left(\bmod \frac{n_{1}}{3}\right), y \equiv u s h_{1}\left(\bmod n_{1}\right)$ and $r y-s x=\frac{n_{1}}{3}$. Since $x \equiv u r h_{1}\left(\bmod \frac{n_{1}}{3}\right)$ and $y \equiv u s h_{1}\left(\bmod n_{1}\right)$, there exist integers $b, d$ such that $x=u r h_{1}+b \frac{n_{1}}{3}$ and $y=u s h_{1}+d n_{1}$. Consider the matrix $S=\left(\begin{array}{cc}3 r & b / h \\ 3 s h & 3 d\end{array}\right)$. As $r y-s x=\frac{n_{1}}{3}$, it is obtained that $3 r d-b s=1$. This yields $S \in \Gamma_{B}(N)$. One can easily verify that $S\left(\frac{1}{0}\right)=\frac{r}{s h}$ and $S\left(\frac{u}{n}\right)=\frac{x}{y h}$. Thus $\frac{r}{s h} \rightarrow \frac{x}{y h}$ is an edge in $G_{u, n}$.

III) The proof of this case follows from Lemma 4.1.

Theorem 4.3. $G_{u, n}$ contains no triangle.

Proof. Suppose in contrary, that $G_{u, n}$ contains a triangle. By the edge conditions (Theorem 4.2) and the transitivity, if $3 \mid n_{1}$, then the triangle is written as

$$
\frac{1}{0} \rightarrow \frac{u}{n} \rightarrow \frac{x}{y} \rightarrow \frac{1}{0}
$$

where all vertices are even; and if $3 \nmid n_{1}$, then it is impossible to write a triangle as in equation (4.3). Thus, we have only the triangle (4.3). Now, since $\frac{u}{n}$ is an even vertex, we can write it as $\frac{u h_{1}}{n_{1} h}$. Therefore, the triangle takes the form

$$
\frac{1}{0} \rightarrow \frac{u h_{1}}{n_{1} h} \rightarrow \frac{x}{y} \rightarrow \frac{1}{0}
$$

For the edge $\frac{x}{y} \rightarrow \frac{1}{0}$, since $\frac{x}{y}$ is an even vertex, there exists an integer $y^{\prime}$ such that $y=3 y^{\prime} h$. By part $I$ of Theorem $4.2(i)$, we obtain that $y^{\prime}=\frac{n_{1}}{3}$.

Now, by rewriting the triangle, we have

$$
\frac{1}{0} \rightarrow \frac{u h_{1}}{n_{1} h} \rightarrow \frac{x}{n_{1} h} \rightarrow \frac{1}{0}
$$

For the edge $\frac{u h_{1}}{n_{1} h} \rightarrow \frac{x}{n_{1} h}$, by $I$ of Theorem $4.2(i)$, we obtain that $x=u h_{1} \pm 1$ and $x \equiv \pm u^{2} h_{1}^{2}\left(\bmod n_{1}\right)$. These two expressions yield $u^{2} h_{1}^{2} \pm u h_{1}+1 \equiv 0\left(\bmod n_{1}\right)$. As $3 \mid n_{1}$, this congruence states that $3 \mid u^{2} h_{1}^{2} \pm u h_{1}+1$, giving that $3 \nmid u h_{1}$ and $3 \nmid u h_{1} \pm 1$. This is a contradiction. Thus, the proof follows.

Theorem 4.4. Let $\frac{u}{n} \in \widehat{\mathbb{Q}},(n, h)=t$ and $n=n_{1} t, h=h_{1} t$ then the followings hold:

i) If $3 \mid n_{1}$, then $G_{u, n}$ contains a hexagon if and only if $u^{2} h_{1}^{2} \pm 2 u h_{1}+1 \equiv 0\left(\bmod n_{1}\right)$.

ii) If $3 \nmid n_{1}$, then $G_{u, n}$ contains a hexagon if and only if $3 u^{2} h_{1}^{2} \pm 3 u h_{1}+1 \equiv 0\left(\bmod n_{1}\right)$.

Proof. i) Let $3 \mid n_{1}$. Assume that $G_{u, n}$ contains a hexagon. By transitivity, and part $I$ of Theorem $4.2(i)$, this hexagon is of the form

$$
\frac{1}{0} \rightarrow \frac{u}{n} \leq \frac{x_{1}}{y_{1}} \leq \frac{x_{2}}{y_{2}} \leq \frac{x_{3}}{y_{3}} \leq \frac{x_{4}}{y_{4}} \rightarrow \frac{1}{0}
$$

where all of the vertices are even. Since $\frac{u}{n}$ is an even vertex, we write it as $\frac{u h_{1}}{3 \frac{n_{1}}{3} h}$. Also, since all of the other vertices are even, there are integers $k_{i}, i=1,2,3,4 \mathrm{such}$ that $y_{i}=k_{i} n_{1} h$. Thus, the hexagon is of the form 


$$
\frac{1}{0} \rightarrow \frac{u h_{1}}{n_{1} h} \leq \frac{x_{1}}{k_{1} n_{1} h} \leq \frac{x_{2}}{k_{2} n_{1} h} \leq \frac{x_{3}}{k_{3} n_{1} h} \leq \frac{x_{4}}{k_{4} n_{1} h} \rightarrow \frac{1}{0} .
$$

For the second edge, by part $I$ of Theorem $4.2(i)$, we get $x_{1}=u h_{1} k_{1}+1$ and $x_{1} \equiv u^{2} h_{1}^{2}\left(\bmod n_{1}\right)$. Hence,

$$
u^{2} h_{1}^{2}+u h_{1} k_{1}+1 \equiv 0\left(\bmod n_{1}\right) .
$$

Also for the last edge, by part $I$ of Theorem $4.2(i)$ we obtain that $1 \equiv$ $-u x_{4} h_{1}\left(\bmod n_{1}\right)$. Using (4.6), we have

$$
x_{4} \equiv u h_{1}+k_{1}\left(\bmod n_{1}\right) .
$$

Now, consider the element

$$
T=\left(\begin{array}{cc}
-u h_{1} & \frac{u^{2} h_{1}^{2}+u h_{1} k_{1}+1}{n_{1} h} \\
-n_{1} h & u h_{1}+k_{1}
\end{array}\right) \in \Gamma_{B}(N) .
$$

It is clear that $T^{2}(\infty)=\frac{x_{1}}{k_{1} n_{1} h}$. One can easily show also that

$$
\begin{aligned}
& T^{3}(\infty)=\frac{\left(k_{1}^{2}-1\right) u h_{1}+k_{1}}{\left(k_{1}^{2}-1\right) n_{1} h}=\frac{x_{2}}{k_{1} n_{1} h}, \\
& T^{4}(\infty)=\frac{\left(k_{1}^{2}-2\right) u h_{1} k_{1}+k_{1}^{2}-1}{\left(k_{1}^{2}-2\right) k_{1} n_{1} h}=\frac{x_{3}}{k_{3} n_{1} h} .
\end{aligned}
$$

Finally, for the last edge, using part $I$ of Theorem $4.2(i)$, we have $k_{4}=1$. Using the edge $\frac{x_{3}}{k_{3} n_{1} h} \leq \frac{x_{4}}{n_{1} h}$, part $I$ of Theorem $4.2(i)$ and the equality

$$
\frac{\left(k_{1}^{2}-2\right) u h_{1} k_{1}+k_{1}^{2}-1}{\left(k_{1}^{2}-2\right) k_{1} n_{1} h}=\frac{x_{3}}{k_{3} n_{1} h},
$$

we have $\left(k_{1}^{2}-2\right) u h_{1}-x_{4}=-k_{1}$. This yields $k_{1}^{2}-2 \mid k_{1}$. Since $k_{1}$ is a positive integer, $k_{1}=1$ or $k_{1}=2$. But if $k_{1}=1$, then the circuit is not a hexagon. Thus we have $k_{1}=2$.

Consequently, substituting $k_{1}=2$ in (4.6), we obtain that $u^{2} h_{1}^{2}+2 u h_{1}+1 \equiv$ $0\left(\bmod n_{1}\right)$.

If the circuit is decreasing, it is obtained similarly that $u^{2} h_{1}^{2}-2 u h_{1}+1 \equiv$ $0\left(\bmod n_{1}\right)$.

Conversely, assume that $u^{2} h_{1}^{2} \pm 2 u h_{1}+1 \equiv 0\left(\bmod n_{1}\right)$. Using Theorem 4.2 , it follows that

$$
\frac{1}{0} \rightarrow \frac{u h_{1}}{n_{1} h} \rightarrow \frac{2 u h_{1} \pm 1}{2 n_{1} h} \rightarrow \frac{3 u h_{1} \pm 2}{3 n_{1} h} \rightarrow \frac{4 u h_{1} \pm 3}{4 n_{1} h} \rightarrow \frac{u h_{1} \pm 1}{n_{1} h} \rightarrow \frac{1}{0}
$$

is a hexagon in $G_{u, n}$.

ii) Let $3 \nmid n_{1}$. First assume that $G_{u, n}$ contains a quadrilateral. By transitivity, and part $I I$ of Theorem $4.2(i i)$, this quadrilateral is of the form

$$
\frac{1}{0} \rightarrow \frac{u h_{1}}{n_{1} h} \leq \frac{x_{1}}{3 k_{1} n_{1} h} \leq \frac{x_{2}}{k_{2} n_{1} h} \leq \frac{x_{3}}{3 k_{3} n_{1} h} \leq \frac{x_{4}}{k_{4} n_{1} h} \rightarrow \frac{1}{0},
$$

where $\frac{x_{1}}{3 k_{1} n_{1} h}$ and $\frac{x_{3}}{3 k_{3} n_{1} h}$ are even vertices, and, $\frac{x_{2}}{k_{2} n_{1} h}$ and $\frac{x_{4}}{k_{4} n_{1} h}$ are odd vertices. Thus, $3 \nmid x_{1}, x_{3}$ and $3 \nmid k_{2}, k_{4}$. 
For the second edge, by part $I$ of Theorem $4.2(i i)$, we get $x_{1}=3 u h_{1} k_{1}+1$ and $x_{1} \equiv 3 u^{2} h_{1}^{2}\left(\bmod n_{1}\right)$. Hence,

$$
3 u^{2} h_{1}^{2}+3 u h_{1} k_{1}+1 \equiv 0\left(\bmod n_{1}\right) .
$$

Also for the last edge, by part $I$ of Theorem 4.2 (ii) we obtain that $1 \equiv$ $-3 u x_{4} h_{1}\left(\bmod n_{1}\right)$. Using (4.10), we have

$$
x_{4} \equiv u h_{1}+k_{1}\left(\bmod n_{1}\right)
$$

Now, consider the element

$$
T=\left(\begin{array}{cc}
-3 u h_{1} & \frac{3 u^{2} h_{1}^{2}+3 u h_{1} k_{1}+1}{n_{1} h} \\
-3 n_{1} h & 3\left(u h_{1}+k_{1}\right)
\end{array}\right) \in \Gamma_{B}(N) .
$$

It is clear that $T^{2}(\infty)=\frac{x_{1}}{3 k_{1} n_{1} h}$. One can easily show also that

$$
\begin{aligned}
T^{3}(\infty) & =\frac{\left(3 k_{1}^{2}-1\right) u h_{1}+k_{1}}{\left(3 k_{1}^{2}-1\right) n_{1} h}=\frac{x_{2}}{k_{1} n_{1} h} \\
T^{4}(\infty) & =\frac{3\left(3 k_{1}^{2}-2\right) u h_{1} k_{1}+3 k_{1}^{2}-1}{3\left(3 k_{1}^{2}-2\right) k_{1} n_{1} h}=\frac{x_{3}}{k_{3} n_{1} h} .
\end{aligned}
$$

Finally, for the last edge, by using part $I$ of Theorem $4.2(i i)$, we have $k_{4}=1$. By using the edge $\frac{x_{3}}{3 k_{3} n_{1} h} \leq \frac{x_{4}}{n_{1} h}$, part II of Theorem $4.2(i i)$ and the equality

$$
\frac{3\left(3 k_{1}^{2}-2\right) u h_{1} k_{1}+3 k_{1}^{2}-1}{\left(3 k_{1}^{2}-2\right) k_{1} n_{1} h}=\frac{x_{3}}{3 k_{3} n_{1} h},
$$

we have $\left(3 k_{1}^{2}-2\right)\left(u h_{1}-x_{4}\right)=k_{1}$. This yields $3 k_{1}^{2}-2 \mid k_{1}$. Since $k_{1}$ is a positive integer, $k_{1}=1$.

Consequently, by substituting $k_{1}=1$ in (4.10), we obtain that $3 u^{2} h_{1}^{2}+3 u h_{1}+1 \equiv$ $0\left(\bmod n_{1}\right)$.

If the circuit is decreasing, we obtain that similarly that $3 u^{2} h_{1}^{2}-3 u h_{1}+1 \equiv$ $0\left(\bmod n_{1}\right)$.

Conversely, assume that $3 u^{2} h_{1}^{2} \pm 3 u h_{1}+1 \equiv 0\left(\bmod n_{1}\right)$. By Theorem 4.2 , it follows that

$$
\frac{1}{0} \rightarrow \frac{u h_{1}}{n_{1} h} \rightarrow \frac{3 u h_{1} \pm 1}{3 n_{1} h} \rightarrow \frac{2 u h_{1} \pm 1}{2 n_{1} h} \rightarrow \frac{3 u h_{1} \pm 2}{3 n_{1} h} \rightarrow \frac{u h_{1} \pm 1}{n_{1} h} \rightarrow \frac{1}{0}
$$

is a hexagon in $G_{u, n}$. 


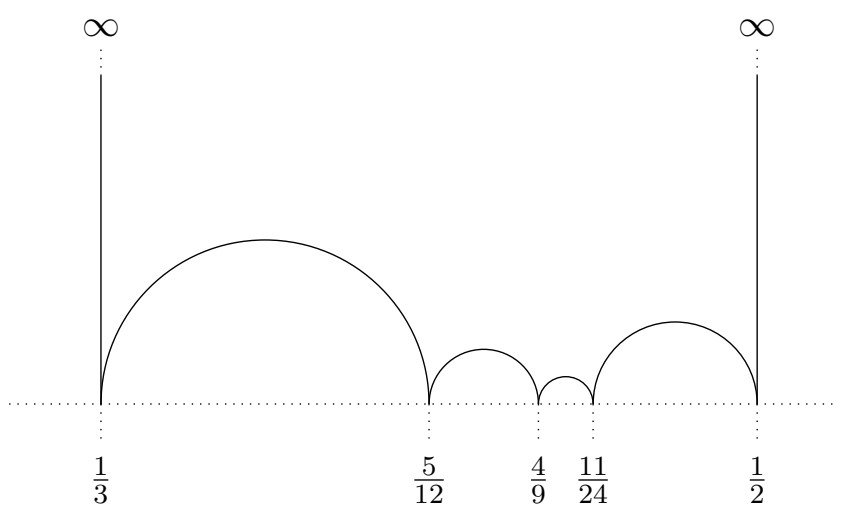

Figure 1. A hexagon in $G_{1,3}$

Example 4.5. A hexagon in $G_{1,3}$ is as follows (see Fig 1)

$$
\frac{1}{0} \rightarrow \frac{1}{2} \rightarrow \frac{5}{12} \rightarrow \frac{4}{9} \rightarrow \frac{11}{24} \rightarrow \frac{1}{2} \rightarrow \frac{1}{0} .
$$

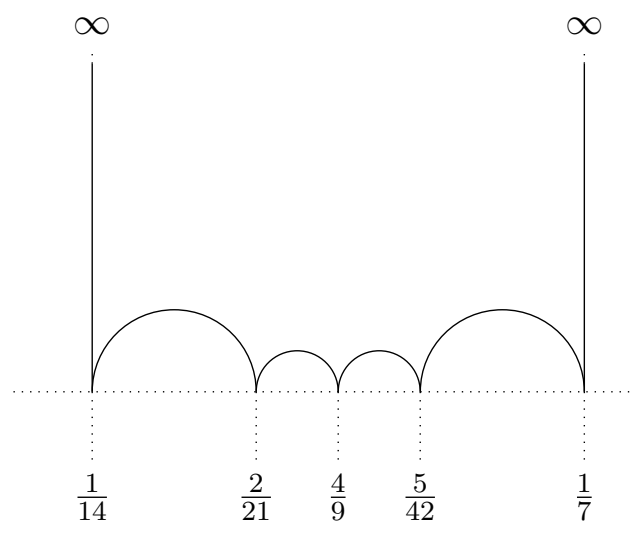

Figure 2. A hexagon in $G_{1,7}$

Example 4.6. A hexagon in $G_{1,7}$ is as follows (see Fig 2)

$$
\frac{1}{0} \rightarrow \frac{1}{7} \rightarrow \frac{5}{42} \rightarrow \frac{3}{28} \rightarrow \frac{2}{21} \rightarrow \frac{1}{14} \rightarrow \frac{1}{0} .
$$

Acknowledgment. The authors would like to thank the anonymous reviewer for his/her valuable suggestions, which greatly improved the earlier version of this paper.

\section{References}

[1] M. Akbaş and D. Singerman, The signature of the normalizer of $\Gamma_{0}(N)$ in $P S L(2, R)$, London Math. Soc. 165, 77-86, 1992.

[2] N.L. Biggs and A.T. White, Permutation groups and combinatorial structures, London Math. Soc. Lec. Not. Ser., 33rd ed. CUP, Cambridge, 1979.

[3] I.N. Cangül and D. Singerman, Normal subgroups of Hecke groups and regular maps, Math. Proc. Camb. Phil. Soc. 123, 59-74, 1998.

[4] K.S. Chua and M.L Lang, Congruence subgroups associated to the monster, Experiment. Math. 13 (3), 343-360, 2004.

[5] J.H. Conway and S.P. Norton, Monstrous Moonshine, Bull. London Math. Soc. 11, 308-339, 1977. 
[6] H.S.M. Coxeter and W.O.F. Moser, Generators and Relations for Discrete Groups, 4th ed. Springer-Verlag, 1984.

[7] H.M. Farkas and I. Kra, Theta constants, Riemann surfaces and the modular group, Graduate Texts in Mathematics, 37, AMS, 2001.

[8] B.Ö. Güler, M. Beşenk and S. Kader, On congruence equations arising from suborbital graphs, Turk. J. Math. 43 (5), 2396-2404, 2019.

[9] I. Ivrissimtzis, D. Singerman and J. Strudwick, From farey fractions to the Klein quartic and beyond, Ars Math. Comtemp. 20 (1), 37-50, 2021.

[10] G.A. Jones and D. Singerman, Theory of maps on orientable surfaces, Proc. London Math. Soc. 37 (3), 273-307, 1978.

[11] I. Ivrissimtzis and D. Singerman, Regular maps and principal congruence subgroups of Hecke groups, Eur. J. Comb. 26, 437-456, 2005.

[12] G.A. Jones and D. Singerman, Complex Functions, an Algebraic and Geometric Viewpoint, CUP, 1987.

[13] S. Kader, Circuits in suborbital graphs for the normalizer, Graphs Combin. 33 (6), 1531-1542, 2017.

[14] C. Maclachlan, Groups of units of zero ternary quadratic forms, Proc. Royal Soc. 88(A), 141-157, Edinburgh, 1981.

[15] C.C. Sims, Graphs and finite permutation groups, Math. Z. 95, 76-86, 1967.

[16] D. Singerman, Universal tessellations, Rev. Mat. Univ. Complut. 1, 111-123, 1988.

[17] D. Singerman and J. Strudwick, Petrie polygons, Fibonacci sequences and Farey maps, Ars Math. Contemp. 10 (2), 349-357, 2016.

[18] D. Singerman and J. Strudwick, The Farey maps modulo n, Acta Math. Univ. Comen. 89 (1), 39-52, 2020.

[19] J. Siran, How symmetric can maps on surfaces be?, Surveys in Combinatorics, 161238, London Math. Soc. Lec. Not. Ser. 409, CUP, Cambridge, 2013.

[20] N. Yazıcı Gözütok, U. Gözütok and B.Ö. Güler, Maps corresponding to the subgroups $\Gamma_{0}(N)$ of the modular group, Graphs Combin. 35 (6), 1695-1705, 2019.

[21] N. Yazıcı Gözütok and B.O. Güler, Quadrilateral cell graphs of the normalizer with signature $(2,4, \infty)$, Stud. Sci. Math. Hung. 57 (3), 408-425, 2020. 